\title{
Paradoxien und Antinomien *
}

\section{Stolpersteine auf dem Weg zur Wahrheit}

\author{
Das sind die Weisen, \\ die durch den Irrtum zur Wahrheit reisen. \\ Die beim Irrtum beharren, \\ das sind die Narren. \\ Friedrich Rückert
}

\section{Was ist paradox?}

\begin{abstract}
Paradox ist, wenn ein Vater seinen Sohn unverwandt anstarrt, wenn ein Arzt kalte Umschläge warm empfiehlt, wenn jemand ein eingefleischter Vegetarier ist, wenn eine Kuh einen anstiert, wenn ein Kahlkopf sich die Haare rauft, wenn ein Lokführer keinen Zug vertragen kann, wenn ein Förster keine Schonung kennt, wenn ein Goethedenkmal durch die Bäume schillert, wenn ein Onkel seinen Neffen vernichtet.
\end{abstract}

Selbst wenn man diese Beispiele amüsant findet, so liefert doch keines von ihnen eine genauere Bestimmung für „paradox“. Wie der Logiker weiß, reichen Beispiele allein nicht aus, um etwas zu definieren, auch wenn sie aus didaktischen Gründen unverzichtbar sind. Wir brauchen eine Definition.

In diesem, wenn auch nicht in jedem Falle kann uns die Etymologie weiterhelfen. Im Griechischen bedeutet $\pi \alpha \varrho \alpha ́$ (pará, mit Akkusativ) ,an ... vorbei“, „entgegen“. (Parapsychologie ist also eine Art Nebenpsychologie.) Und $\delta o ́ \xi \alpha$ (dóxa) ist „Meinung“, „Glaube“, „Erwartung“. Ein Paradoxon ist also ein Sachverhalt, der der Erwartung zuwiderläuft. Und eine Aussage, die einen paradoxen Sachverhalt, etwas Paradoxes beschreibt, nennen wir ebenfalls paradox oder eine „Paradoxie“.

* Festvortrag gehalten am 27. November 1987 in Gießen anläßlich der Akademischen Jahresfeier der Justus-Liebig-Universität (gekürzte Fassung).
Der Begriff „Antinomie“ ist etwas schwerer zu bestimmen; er hat eine stärkere Wandlung mitgemacht. ${ }^{1}$ Im 17 . Jahrhundert steht er für den Widerstreit von Gesetzen (contrarietas legum); so kann es zwischen Naturrecht und positivem Recht $\mathrm{zu}$ einer Antinomie kommen. Gelegentlich werden auch Widersprüche in der Bibel als Antinomien angesehen. Und bei Kant bezeichnet „die Antinomie der Vernunft" die Tatsache, daß sich das Denken in Widersprüche verwickelt, wenn es seine Zuständigkeiten überschreitet. In diesem Sinne gibt es dann nur eine Antinomie der reinen Vernunft. Da Kant jedoch vier solcher Widersprüche aufzählt und diese gelegentlich auch Antinomien nennt, hat es sich eingebürgert, von Kantischen Antinomien auch im Plural zu sprechen.

Antinomien bilden also eine bestimmte Klasse von Widersprüchen, nämlich solche, bei denen sich beide Seiten (These und Antithese) allem Anschein nach gleich gut begründen lassen. Die Entdekkung einer Antinomie belehrt uns dann zwar darüber, daß in den Voraussetzungen etwas nicht stimmt (sonst hätte es keinen Widerspruch gegeben); sie macht jedoch nicht auch schon deutlich, wo der Fehler liegt und welche der beiden anscheinend so trefflich begründeten Seiten schließlich doch aufgegeben werden muß. Antinomien können überall auftreten: bei beschreibenden Sätzen (ist die Welt endlich oder unendlich?), bei normativen Sätzen (darf man töten?), bei Wertungen (ist Freiheit wichtiger als Gleichheit?), bei Konventionen (darf man Fisch mit dem Messer essen?), bei Schlußregeln (gilt et- 
was, was für jeden gilt, damit auch schon für alle?). Bei Normen und Werten ist "Antinomie" nahezu bedeutungsgleich mit „Konflikt“"; macht man überhaupt einen Unterschied, so wird man ,Antinomien" wieder nur solche Konflikte nennen, bei denen beide Seiten starke Argumente, Überzeugungen oder Plausibilitäten für sich haben.

Am wichtigsten und folgenreichsten sind Antinomien dort, wo sie am wenigsten erwartet werden und am wenigsten erwünscht sind, also vor allem in den Grundlagen einer Disziplin. Wenn Gesetze einander widersprechen, woran soll man sich dann noch halten? Wenn die Vernunft auf Widersprüche führt, (wie) kann man diesen mit Vernunft entgehen? Wenn es sogar in der Mathematik, der Königin der Wissenschaften, solche Inkonsistenzen gibt, wie soll man sich dann gegen solche Fehler schützen? Und wenn nun gar in der Logik, in der seit Jahrtausenden vermeintlich unanfechtbaren Lehre vom richtigen Schließen, Widersprüche auftauchen, wenn also noch unser elementarstes Handwerkszeug unbrauchbar ist, woher sollen dann Zuverlässigkeit und Sicherheit überhaupt noch kommen?

Im Englischen ist "antinomy" wenig gebräuchlich. Selbst die um 1900 entdeckten Antinomien in Logik und Mathematik heißen dort einfach "paradoxes“. Nur in der metalogischen und metamathematischen Fachsprache wird - zur schärferen Ausgrenzung - gelegentlich von ,,antinomies" gesprochen. Auch im Deutschen ist die Verwendungsweise nicht genau festgelegt. Hat man jedoch einerseits zwei unterschiedliche Sachverhalte (oder Objektklassen) und andererseits zwei dazu passende Wörter, so sollte man sich diesen sprachlichen Reichtum auch zunutze machen. Dadurch fällt es dem Juristen leicht, Besitz (worüber man verfügt) von Eigentum (was einem gehört) abzugrenzen; ähnlich unterscheidet der Botaniker Dornen und Stacheln, der Physiker Kraft und Energie. So ist es auch zweckmäßig, Paradoxien von Antinomien zu unterscheiden.

Wir werden „Paradoxie“ als den weiteren, „Antinomie“" als den engeren Begriff auffassen. Alles, was der Erwartung zuwiderläuft, insbesondere etwas, das sich unerwartet als wahr (oder unerwartet als falsch) erweist, heißt dabei paradox. Dagegen bezeichnen wir nur solche Widersprüche als antinomisch, deren beide Seiten gleich gute Begründungen haben (zu haben scheinen). Alle Antinomien sind danach auch Paradoxien; jedoch kann es durchaus Paradoxien geben, die nicht antinomisch sind. In dieser Präzisierung kann sich auch herausstellen, daß so manche vermeintliche Antinomie gar nicht antinomisch, sondern "nur" paradox ist; und diese Entdeckung mag wiederum manchen so überraschen, daß er darin ein weiteres Paradoxon, eine Meta-Paradoxie, zu sehen bereit ist.

\section{Der „Lügner" - eine Antinomie}

Das bekannteste Beispiel für eine Antinomie und jedenfalls eines der ältesten ist die Lügner-Antinomie „Ich lüge jetzt.“ Will man Komplikationen, insbesondere das problematische Element der Lüge (der $b e$ wußten Unwahrheit) vermeiden, so formuliert man noch einfacher:

$$
\text { „Dieser Satz ist falsch." (S1) }
$$

Ist das wahr, so ist wahr, was der Satz sagt. Er sagt aber, er sei falsch; also ist er falsch. Ist er also falsch? Nehmen wir einmal an, er sei tatsächlich falsch. Dann muß das, was er sagt, falsch sein. Nun sagt er, er sei falsch. Wenn es also wirklich falsch ist, daß er falsch ist, dann kann er wieder nur wahr sein. Also gilt: Wenn der Satz wahr ist, so ist er falsch; und ist er falsch, so ist er wahr. Er ist also wahr dann 
und nur dann (genau dann), wenn er falsch ist. Das ist offensichtlich ein Widerspruch, und wegen der völligen Symmetrie der Argumentation ist es sogar eine Antinomie.

Wie kommen wir aus dieser Antinomie heraus? Können wir den Widerspruch auflösen, beseitigen, vielleicht verhindern? Betrachten wir die Argumentation noch einmal genauer: Aus der Annahme, (S1) sei wahr, folgt offenbar, daß (S1) falsch ist. Wahr kann (S1) also nicht sein. Aus der Annahme, (S1) sei falsch, folgt dagegen, daß (S1) wahr ist. Falsch kann (S1) also ebenfalls nicht sein. (S1) ist demnach weder wahr noch falsch. Aber was ist (S1) dann? Kann es Aussagen geben, die weder wahr noch falsch sind?

Nach der klassischen Logik ist eine (deskriptive) Aussage ein sprachliches Gebilde, von dem es sinnvoll ist zu sagen, es sei wahr oder falsch. (Ob die Aussage dabei wirklich wahr ist und wie man das herausfindet, spielt dabei - nach klassischer Auffassung - keine Rolle.) Andere sprachliche Gebilde wie Befehle, Verbote oder Fragen können gar nicht wahr oder falsch sein, sind also auch keine (deskriptiven) Aussagen. Wenn Aussagen gerade darüber definiert sind, daß sie einen Wahrheitswert haben, dann kann es Aussagen ohne Wahrheitswert überhaupt nicht geben. Ein sprachliches Gebilde, das weder wahr noch falsch ist, kann also gar keine Aussage sein. Der Ausdruck ,Dieser Satz ist falsch.", sieht zwar zunächst so aus wie eine Aussage, ist aber keine. Es ist zwar ein sprachliches Gebilde, aber kein Satz (oder, falls man die Terminologie etwas toleranter gewählt hat, wenigstens kein sinnvoller oder kein zulässiger Satz).

Offenbar sind wir dadurch in eine Antinomie geraten, daß wir ein sprachliches Gebilde, das aussieht wie ein Satz, vorschnell als Satz anerkannt haben. Wir lernen dar- aus, daß nicht alles, was wie ein Satz aussieht, wirklich ein Satz ist. Und wir vermeiden die Antinomie, indem wir diesen und ähnlichen antinomischen Gebilden die Anerkennung als Satz verweigern, sie gar nicht erst als Sätze ansehen, auch wenn sie sich noch so artig als Sätze, noch so ,satz-artig“ geben.

Für die Lügner-Antinomie gibt es hübsche Einkleidungen. Am besten gefällt mir

\section{„Diser Sats enthält drei Fehler.“ (S2)}

Zunächst fallen einem nur die Rechtschreibfehler auf. Kommt man jedoch auf die Idee, die Fehler nachzuzählen, so findet man nur zwei. Es ist aber von drei „Fehlern" die Rede. Hat sich der Autor vielleicht verzählt? Jedenfalls hat er einen Fehler gemacht, einen inhaltlichen. Ach, da ist er ja nun doch, der gesuchte dritte Fehler! Der Satz enthält tatsächlich drei Fehler, zwei orthographische und einen inhaltlichen. Ist er also wahr? Wenn er aber wahr ist, wo ist dann eigentlich der dritte Fehler? Dann enthält er ja doch nur die zwei Rechtschreibfehler und keinen inhaltlichen. Aber dann wird er eben dadurch wieder falsch usw. Wieder finden wir das typische Hin und Her zwischen Wahr und Falsch, den für Antinomien charakteristischen ,Zickzack" der Wahrheitswerte.

Für den Nachweis, daß es sich auch hier nur um eine besonders ausgeschmückte Version der Lügner-Antinomie handelt, empfiehlt es sich, die Rechtschreibfehler schrittweise $z u$ beseitigen und dabei die Zählung entsprechend zu erniedrigen:

\section{„Dieser Sats enthält zwei Fehler.“ \\ „Dieser Satz enthält einen Fehler."}

Die letzte Formulierung ist dann nichts anderes als der Lügner (S1). Umgekehrt kann man die Zahl der Fehler auch beliebig erhöhen. 


\section{Der „Kreter" - nur eine Paradoxie}

Häufig wird die Lügner-Antinomie in noch anderer Form dargestellt:

„Epimenides, der Kreter, sagt:

Alle Kreter lügen immer." (S3)

Einen Kreter Epimenides hat es um 600 v. Ch. wohl wirklich gegeben. Authentisches ist von ihm nicht überliefert. Die Legende will jedoch, daß er 57 Jahre in einer Grotte geschlafen oder dies wenigstens behauptet habe. Auch anderen Kretern wird schon im Altertum Schlechtes nachgesagt, so von Livius oder Plutarch. Daß sie Lügner seien, behaupten Polybios, Diogenianus, Psellus und Suidas. Und sogar der Apostel Paulus schreibt in seinem Brief an Titus:

Es hat einer aus ihnen gesagt, ihr eigner Prophet: „Die Kreter sind immer Lügner, böse Tiere und faule Bäuche." Dies Zeugnis ist wahr.

Es lag gewiß nicht in der Absicht des Apostels, auf die hier drohende Paradoxie aufmerksam zu machen. (Vielmehr gibt er seinem Vertreter Titus Anweisungen, wie er die besonderen Probleme Kretas anzupacken habe.) Da Paulus selbst kein Kreter ist, ergibt sich auch aus seinem letzten Satz kein zusätzliches Problem. (Wäre er einer, so gäbe es allerdings ganz schöne Verwicklungen.) Falls er aber mit ,ihrem eignen Propheten" Epimenides meint, dann wird seine Formulierung identisch mit (S3). Handelt es sich hier tatsächlich um eine Antinomie? Wir können das leicht nachprüfen. Nehmen wir an, die Aussage des Epimenides, also (S3), sei wahr. Dann sind alle Kreter und somit auch er selbst notorische Lügner. Also lügt er auch jetzt, und es ist nicht wahr, daß alle Kreter immer lügen. Wahr kann die Aussage des Epimenides also nicht sein. Kann sie falsch sein? Angenommen, sie sei falsch; dann sagen manche Kreter manchmal (sagt wenigstens ein Kreter einmal) die Wahrheit. Daraus folgt jedoch nicht, $\mathrm{da} ß$ gerade Epimenides ausgerechnet jetzt die Wahrheit sagt. (Das würde, wie schon Eubulides bemerkt hat, nur dann folgen, wenn Epimenides der einzige Kreter überhaupt wäre und nur diesen einen Satz von sich gäbe. Das können wir unter Verweis auf die Tatsachen ausschließen. Es ist aber bemerkenswert - ein neues Paradoxon?-, daß für die Frage, ob etwas antinomisch ist oder nicht, Tatsachen eine Rolle spielen können.)

Die Annahme, (S3) sei falsch, führt also nicht zu einem Widerspruch. Wir haben somit keine Zickzack-Struktur (sondern nur einen ,Zick"); (S3) ist nicht antinomisch, sondern "nur" falsch. Der Selbstbezug und die unerwartete Selbstwiderlegung geben (S3) jedoch unzweifelhaft paradoxen Charakter.

Viele Formulierungen weisen einen solchen Selbstbezug auf, stellen sich in Frage oder widerlegen sich selbst und sind eben dadurch paradox. Hierfür nun noch einige Beispiele.

\section{Innere Ungereimtheiten}

Aberglaube bringt Unglück; dagegen hilft nur dreimaliges Klopfen auf Holz.

Ich bin "Skorpion"; deshalb halte ich nichts von Astrologie.

Gott sei's gedankt, ich bin immer noch Atheist. (Buñuel)

Es ist modern, altmodisch zu sein.

Auch die Nostalgie ist nicht mehr das, was sie einmal war.

Nichts ist ewig außer dem Wechsel.

Sicheres Wissen gibt es mit Sicherheit nicht.

Hat Hans wirklich Wahnvorstellungen? Nein, das Problem ist, daß er sich einbildet, er habe welche. (Smullyan)

Dies ist ein Aprilscherz.

Dieser Satz erscheint antinomisch, ist es aber gar nicht. 
Auf Verlangen muß der Beschuldigte über seine Rechte aufgeklärt werden.

Nur ein einziges Dogma kann und muß im Monon existieren, nämlich daß es keine Dogmen geben darf. (Bresch)

Ein Satz, der nur wahrscheinlich ist, ist wahrscheinlich falsch. (Descartes)

Keine Regel ohne Ausnahme.

Die Geschichte lehrt, daß die Menschen aus der Geschichte nichts lernen. (Hegel)

Dieser Satz kann niemals bewiesen werden. (Gödel)

Wenn Gott allmächtig ist, kann er dann einen Stein schaffen, der so schwer ist, daß er ihn selbst nicht heben kann? (Pascal)

Alles dauert länger, als man denkt, selbst dann, wenn man dies bereits berücksichtigt hat. (Murphy/Hofstadter)

Philosophie ist der Mißbrauch einer Sprache, die eigens zu diesem $Z$ weck erfunden wurde.

\section{Paradoxien für die Forschung}

Widersprüche lassen wir nicht auf sich beruhen. Sie zeigen, daß etwas nicht stimmt. Intuitive Erwartungen, plausible Annahmen, elegante Formulierungen, gutgemeinte Vorschriften, einleuchtende Regeln, abstrakte Theorien - sie alle können Fehler enthalten. Ohne Paradoxie wird ein Fehler vielleicht nie entdeckt. Und deshalb auch nicht beseitigt. Wer weiß, welchen Schaden er dann noch anrichtet? Schon die antiken Logiker haben nämlich festgestellt, daß logische Widersprüche jeden beliebigen Satz abzuleiten gestatten: „Ex contradictione quodlibet (sequitur); aus einem Widerspruch (folgt) Beliebiges.", lautet deshalb auch ein Grundsatz der klassischen Logik. Und so ungemein ergiebig ein System zunächst ja noch erscheinen mag, aus dem jede gewünschte Aussage herausgeholt werden kann, so enttäuschend ist die Entdeckung, daß zu jeder gewünschten Aussage auch ihr Ge- genteil ableitbar ist, zu jeder wahren Aussage eine falsche, zu jeder These eine Gegenthese. Wer Widersprüche zuläßt, der kann somit Wahrheit oder Geltung überhaupt nicht mehr sinnvoll beanspruchen.

Deshalb darf und wird sich ein Wissenschaftler mit Widersprüchen niemals abfinden. Und da Paradoxien auf Widersprüche verweisen oder solche sogar darstellen, bieten sie den besten Anreiz, das System gründlich zu überdenken und nach Verbesserungen zu suchen.

Die Entdeckung einer Paradoxie oder gar einer Antinomie hat somit immer etwas Zweischneidiges. Daß irgendwo ein Fehler steckt, ist natürlich bedauerlich; daß er nun aber, wenn es ihn schon einmal gibt, gesucht, entdeckt und beseitigt werden kann, das ist - gerade deshalb - um so erfreulicher. Widersprüche entstellen eine Theorie, die so nicht wahr sein kann; und doch ist die Entdeckung eines Widerspruchs ein Glücksfall für die Disziplin, ein unübersehbarer Ansporn, es besser zu machen. Eine solche Entdeckung kann also die Theorie töten und zugleich die Aufmerksamkeit, das Nachdenken, die Diskussion, die Theorienbildung beleben. Viele Fortschritte in der Wissenschaft sind solchen Entdeckungen zu verdanken. Der Physiker John Archibald Wheeler meint sogar, ohne Paradoxien gebe es überhaupt keinen wissenschaftlichen Fortschritt. ${ }^{2}$

Immerhin - Wissenschaft ist Wahrheitssuche. Es gibt zwar keinen Königsweg zur Wahrheit, wohl aber einen Fußweg: Versuch und Irrtumsbeseitigung. Je mehr Irrtümer wir beseitigen können, desto besser sind die Chancen, daß wir die Wahrheit übrigbehalten. Um aber Irrtümer beseitigen zu können, muß man sie erkennen; und um sie zu erkennen, sind Paradoxien ein bewährtes Mittel. Deshalb spielen Paradoxien für die Forschung eine so wichtige Rolle. Betrachten wir ein Beispiel aus der Mathematikgeschichte. 


\section{Die berïhmteste, die Russellsche Antinomie}

Die Russellsche Antinomie entsteht bei dem Versuch, alle Mengen, die sich nicht selbst als Element enthalten, zu einer Menge zusammenzufassen. Nach der klassischen Logik und nach der von Georg Cantor entwickelten Mengenlehre ist diese Operation völlig legitim: Alle Objekte mit einer bestimmten Eigenschaft sollten sich zu einer Menge vereinen lassen, jede beliebige Eigenschaft sollte mengenbildend sein. (Die naive Mengenlehre legt also ein unbeschränktes Komprehensionsprinzip zugrunde.) Der genannte Versuch führt jedoch auf einen Widerspruch. Dies konnte freilich erst entdeckt werden, nachdem Gottlob Frege (1848-1925) die Prinzipien der klassischen Logik scharf formuliert und axiomatisiert hatte.

Noch in der Einleitung zum ersten Band (1893) seines Hauptwerkes "Grundgesetze der Arithmetik" schreibt er selbstbewußt, er wäre widerlegt, wenn jemand ihm nachwiese, daß seine Grundsätze zu offenbar falschen Folgesätzen führten. „Aber das wird keinem gelingen." Ganz anders liest es sich dann zehn Jahre später (1903) im Anhang zum zweiten Band:

Einem wissenschaftlichen Schriftsteller kann kaum etwas Unerwünschteres begegnen, als da $B$ ihm nach Vollendung einer Arbeit eine der Grundlagen seines Baues erschüttert wird. In diese Lage wurde ich durch einen Brief des Herrn Bertrand Russell versetzt, als der Druck dieses Bandes sich seinem Ende näherte.

Frege ist, wie er Russell brieflich gesteht, überrascht, ja bestürzt. Es gelingt ihm auch nicht, die Antinomie zu beseitigen. Nach einem mißglückten Lösungsvorschlag wendet er sich enttäuscht anderen Problemen zu. Die Entdeckung der Russellschen Antinomie empfindet er als persönliche Niederlage. Und doch bemerkt er in seiner spontanen Antwort an Russell ebenso weitsichtig wie großherzig, dessen merkwürdige Entdeckung werde „viel- leicht einen großen Fortschritt zur Folge haben, so unerwünscht sie auf den ersten Blick auch scheinen mag“. Auch damit hat er recht. Er selbst hat diesen Fortschritt zwar nicht erzielt, wohl aber ermöglicht. Erzielt haben ihn neben anderen Russell und Whitehead mit ihrer gigantischen Grundlagenstudie, den „Principia Mathematica". Im Vorwort zum ersten von drei Bänden (1910) meinen sie anerkennend:

In allen logisch-analytischen Fragen verdanken wir das meiste Frege. Wo wir von ihm abweichen, geschieht es meist, weil die Widersprüche zeigten, daß er - wie übrigens alle alten und modernen Logiker - einen Irrtum in seine Voraussetzungen sich hatte einschleichen lassen; ohne die Widersprüche aber wäre es fast unmöglich gewesen, diesen Irrtum aufzudekken.

Noch deutlicher wird Christian Thiel in einer Darstellung und Würdigung von Freges Werk:

Man kann es geradezu als tragische Ironie bezeichnen, da $B$ dieser Widerspruch zwar auch in allen andern damals bekannten logischen Systemen steckte, daß aber erst Freges System mit seinem lückenlosen Aufbau den strengen Nachweis dieses Widerspruchs erlaubte. ${ }^{3}$

Die Russellsche Antinomie hat eine Grundlagenkrise ausgelöst, wie sie in der Mathematik allenfalls die Pythagoreer bei der Entdeckung der irrationalen Zahlen (bzw. inkommensurabler Strecken) hatten erleben müssen. Dabei wurden jedoch mehrere Auswege aus dieser Sackgasse gefunden und eben dadurch auch die von Frege erhofften Fortschritte erzielt. Wir dürfen also festhalten: Ohne (Freges) Präzisierung kein Ableiten von Antinomien, ohne die Antinomien kein Aufdecken der Fehler in den Grundlagen, und ohne diese Entdeckung auch kein Fortschritt.

Antinomien sind wie Steine unter der Wasseroberfläche: Man kann über sie stolpern und untergehen; man kann aber - wenn man sie kennt - auf ihnen auch heil über das Wasser gelangen. 


\section{Was kümmern uns überwundene Paradoxien?}

Paradoxien haben - in geeigneter Verpakkung - hohen Unterhaltungswert. Um den Barbier, der angeblich genau die Männer seines Dorfes rasiert, die sich nicht selbst rasieren, - eine populäre Einkleidung der Russellschen Antinomie entspinnt sich leicht ein angeregtes Gespräch. Daß zwei der anwesenden Personen den gleichen Geburtstag hätten, kann Gegenstand einer spannenden Wette sein. Und wer hätte noch nie darüber gegrübelt, wie die besorgte Mutter ihr Kind vielleicht doch noch von dem scheinheiligen Krokodil zurückbekommen kann? ${ }^{4}$ Außerdem gehören Paradoxien zur Geschichte der Wissenschaft. Gar zu leicht und gar zu häufig wird Wissenschaft (miß)verstanden als ein großes Mosaik, dem Steinchen um Steinchen hinzugefügt wird. Ein solches kumulatives Modell wird nicht erst durch eine verfehlte Wissenschaftstheorie vertreten; es wird auch durch den Aufbau des Unterrichts und unserer Lehrbücher nahegelegt. Gelehrt wird darin selbstverständlich nur Richtiges: Man stellt nur die richtigen Fragen, macht nur taugliche Experimente, sammelt nur brauchbare Ergebnisse und gibt nur richtige Deutungen. Und zu jedem Stück Lehrbuch-Wissen läßt sich anscheinend auch angeben, wem es historisch zu verdanken ist. In Wahrheit wird das kumulative Wissenschaftsmodell weder den historischen Tatsachen gerecht noch bietet es eine rationale Rekonstruktion des Theorienwandels. Auch Irrtümer, Sackgassen, Mißerfolge, Zirkel, Widersprüche, auch Paradoxien und Antinomien sind Teil der Wissenschaftsgeschichte; für den kollektiven Erkenntnisfortschritt sind sie charakteristisch und sogar unverzichtbar. Zugegeben, der Unterhaltung kann auch anderes dienen. Und nicht jeder hat Zeit und Lust, sich mit der Geschichte seiner Disziplin oder des Denkens überhaupt zu befassen. Paradoxien haben aber auch noch andere Aufgaben. Vor allem kann man aus ihnen etwas lernen, auch und gerade aus solchen, die bereits überwunden oder aufgelöst sind und den Forschern eigentlich kein Kopfzerbrechen mehr machen. Der Mathematiker Herbert Meschkowski spricht in diesem Zusammenhang sogar von der „Bildungsfunktion der Paradoxie". ${ }^{5}$ Und weil Paradoxien sowohl unterhaltsam als auch lehrreich sind, eignen sie sich besonders gut für didaktische Zwecke.

Ohne hier nun einen eigenen bildungstheoretischen oder didaktischen Entwurf vorlegen zu wollen, dürfen wir doch wenigstens thesenartig festhalten, was man aus der Beschäftigung mit Paradoxien lernen kann.

- Verallgemeinerungen sind riskant und müssen auf ihre Zulässigkeit geprüft werden. Paradoxien können voreilige Verallgemeinerungen ad absurdum führen. Meschkowski meint sogar, alle Paradoxien entstünden durch unzulässige Verallgemeinerung.

- Unsere Intuition ist fehlbar. Evidenzen sind keine Garantien, Überzeugungen keine Beweise, Bekenntnisse keine Argumente, Autoritäten keine Wahrheitsgaranten.

- Sprache ist eine Haushaltserfindung und zunächst auch nur für den Hausgebrauch tauglich. Sie kann zu Fehldeutungen, Fehlschlüssen, Fehlintuitionen führen, ja verführen. Vom „Offenbarungscharakter" der Sprache sollte man besser gar nicht erst reden.

- Über Bedeutung, Wahrheit, Geltung machen wir häufig stillschweigende Voraussetzungen. Paradoxien können uns helfen, sogar zwingen, solche Annahmen bewußtzumachen, nötigenfalls zu korrigieren. 
- Viele Ideen, Begriffe, Vorstellungen, Intuitionen, Formulierungen sind unscharf und bedürfen einer Präzisierung. Darauf können im Einzelfall - etwa beim Mengenbegriff - Paradoxien besonders drastisch aufmerksam machen.

- Allerdings sind nicht alle Ideen einer solchen Präzisierung fähig. Deshalb können auch übertriebene Präzisionsansprüche zu Paradoxien führen, etwa die Frage: Wie viele Körner bilden einen Haufen? ${ }^{6}$

- Aus einer Antinomie kann es mehrere Auswege geben. Auch in der Wissenschaft können Pluralismus und Toleranz angebracht, können Entschlüsse und Konventionen erforderlich sein.

- Gewohnheiten sind nützlich, bieten aber keine Wahrheitsgarantie. Was alle tun und was schon immer so gemacht wurde, das braucht darum noch nicht richtig $\mathrm{zu}$ sein.

- Auch Denkgewohnheiten sind keine Denknotwendigkeiten.

- Menschen sind fehlbar, selbst die gescheitesten, aufgeklärtesten, scharfsinnigsten. Wir sollten unsere Systeme deshalb fehlertolerant, ja fehlerfreundlich gestalten. Menschliches Versagen kann nie ganz ausgeschlossen, wohl aber durch Sicherheitsspielräume aufgefangen werden.

Die letzte Lehre ist die allgemeinste, und wir wollen - schon um nicht gleich selbst einer unzulässigen Verallgemeinerung schuldig zu werden - keineswegs behaupten, daß diese und andere Lehren sich nur aus Paradoxien ziehen ließen. Viele Wege führen nach Rom und viele zu Bildung; $\mathrm{da} B$ aber Paradoxien einen dieser Wege öffnen, das sollte doch einleuchten. Deshalb wagen wir es auch, hier noch einige paradoxe Formulierungen zusammenzustellen.

\section{Paradoxe Einsichten und Sprüche}

Das Überflüssige ist eine höchst notwendige Sache. (Voltaire)
Ein Parameter ist eine variable Konstante. (Gell-Mann)

Wie gewöhnlich hast Du Dich selbst übertroffen.

Niemals würde ich einem Club beitreten, der bereit wäre, jemanden wie mich aufzunehmen. (Groucho Marx)

Das Immunsystem ist auf das Unerwartete programmiert. (Hilschmann)

Warum bist Du nur immer so hilfsbereit?Ach, weißt Du, altruistisch zu sein, verschafft mir immer so ein tolles Gefühl.

Eine Anekdote ist ein erhellender Bericht über ein Ereignis, das niemals stattgefunden hat. (Eves)

Bildung ist das, was übrigbleibt, wenn man alles vergißt, was man gelernt hat.

Deine Phantasie ist zu mehr fähig, als Du Dir ausmalen kannst. (Aragon)

Was ist der Grund für das Unwissen und die Gleichgültigkeit des Wählers? - Weiß ich nicht, ist mir auch egal! (Paulos)

Komm, wann Du willst; aber sei pünktlich! Keine Toleranz gegenüber den Feinden der Toleranz! (Popper)

Er gab seinen letzten Pfennig für eine Geldbörse aus.

Auch die Mäßigung sollte man nicht ins $E x-$ trem treiben. (Koestler)

Es wird gespart, koste es, was es wolle!

Warum verdaut der Magen sich nicht selbst? (Scientific American 226 [Jan. 1972] 86-93]

Ich wette um zwei Mark, daß ich Ihnen zehn Mark gebe, wenn Sie mir fünf Mark geben!

Sollte diese Nachricht Dich nicht erreichen, so melde Dich!

\section{Kann man Antinomien vermeiden?}

Daß Erfahrungen unseren Erwartungen widersprechen, wird immer wieder vorkommen. Paradoxien in diesem allgemeinen Sinne machen das Leben farbig, die Forschung aufregend, die Wissenschaft 
spannend, den Unterricht lebendig. Das Staunen sei der Anfang aller Philosophie, sagen Platon und Aristoteles, wobei ihnen „Philosophie“" auch das heißt, was wir heute "Wissenschaft" nennen. Auf Paradoxien im Sinne des Überraschenden wollen wir gar nicht verzichten, und deshalb werden wir sie auch nicht zu vermeiden suchen.

Außerdem sind Erwartungen etwas Subjektives; was den einen überrascht, das ist dem anderen bereits vertraut. Sind also Paradoxien ein Bildungselement, so hängt doch ihr paradoxer Charakter auch umgekehrt vom Informations- und Bildungsstand des Betreffenden ab. Es wäre deshalb unmöglich, jemandem alle Überraschungen zu ersparen. Paradoxien grundsätzlich zu vermeiden, ist also weder wünschenswert noch erreichbar.

Anders steht es mit den logischen Widersprüchen, insbesondere mit den Antinomien der Logik, der Semantik, der Mengenlehre, der Mathematik. Sie sind zwar auch lehrreich, aber damit nicht schon unbedingt willkommen. Viele gäben etwas darum, das Auftreten von Widersprüchen verhindern und die Widerspruchsfreiheit ihrer Theorien garantieren zu können.

Dazu wird man zunächst einmal studieren, wie die bereits entdeckten Antinomien entstehen. Sie alle haben zwei gemeinsame Merkmale: Sie zeigen eine bestimmte Kombination aus Rückbezüglichkeit und Verneinung. Der Lügnersatz behauptet von sich selbst, er sei falsch; die Russellsche Menge soll aus Mengen bestehen, die sich selbst nicht als Element enthalten; und bei Begriffen wie "selbstdeskriptiv“ oder "selbstanwendbar" ist der Selbstbezug offensichtlich. ${ }^{7}$

Es liegt deshalb nahe, den Antinomien dadurch auszuweichen, daß man Selbstbezug oder Selbstreferenz grundsätzlich verbietet. Diesen Weg beschreiten Russell und Whitehead, wenn sie in den Principia
Mathematica das Zirkelfehlerprinzip (Circulus-vitiosus-Prinzip) formulieren: Was bereits alle Elemente einer Gesamtheit voraussetzt, kann selbst nicht Element dieser Gesamtheit sein. Diesen Weg geht auch Tarski, wenn er die strenge Unterscheidung von Objekt- und Metasprache fordert und die semantische Geschlossenheit einer Sprachstufe und damit jeden Selbstbezug verbietet. Tatsächlich kann man, wenn man diese Prinzipien befolgt, die üblichen Antinomien nicht mehr erzeugen.

Trotzdem können diese Lösungen nicht restlos befriedigen. Sie leisten nämlich zuviel. Schließlich führen nicht alle Selbstbezüge zu Widersprüchen. Sie sind also nicht alle schädlich oder vitiös, und Unschädliches braucht man eigentlich nicht zu verbieten. In vielen Fällen sind Selbstbezüge nicht nur harmlos, sondern sogar nützlich. Solche fruchtbaren Rückkopplungen kann man virtuose Zirkel nennen. ${ }^{8}$ Das Verbot sämtlicher Selbstbezüge entspricht damit der Amputation eines ganzen Beines, bei dem nur ein Zeh entzündet ist. Man sucht deshalb nach schonenderen Behandlungsmethoden. Solche hat man auch gefunden; es ist jedoch nicht evident, welche von ihnen nun als die natürlichste angesehen werden sollte.

Besonders beunruhigend ist die Tatsache, $\mathrm{da} ß$ man bei diesen Vorschlägen vor weiteren Widersprüchen nicht wirklich geschützt ist: Auch in den gereinigten Systemen könnten eines Tages wieder Antinomien auftauchen. Der Grundlagenforscher fühlt sich wie Herakles im Kampf mit der Lernäischen Schlange, der für jeden abgeschlagenen Kopf neue nachwachsen (können).

Man kann deshalb verstehen, warum David Hilbert (1862-1943) die Forderung aufstellt, die Mathematiker sollten die Widerspruchsfreiheit ihrer Theorien beweisen. Ein solcher Beweis würde garantie- 
ren, daß auch in Zukunft keine Widersprüche mehr auftauchen. Für einige Theorien können solche Widerspruchsfreiheitsbeweise tatsächlich geführt werden. Trotzdem erweist sich Hilberts beweistheoretisches Programm insgesamt als undurchführbar. Kurt Gödel (19061978) gelingt 1931 der Nachweis, daß ein widerspruchsfreies formales System, das wenigstens die Zahlentheorie enthält, mit den Mitteln des Systems - mit „Bordmitteln" sozusagen - nicht als widerspruchsfrei ausgewiesen werden kann. Damit ist Hiberts Traum zerstört: Der Kampf mit dem Drachen „Antinomie“ geht immer noch weiter.

\section{Anmerkungen}

${ }^{1}$ Die Begriffsgeschichte von „Antinomie" behandelt N. Hinske in: J. Ritter (Hrsg.), Historisches Wörterbuch der Philosophie, Band 1, Basel 1971, Sp. 393-396.

2 Wheeler, J.A. A septet of sibyls: aids in the search for truth. American Scientist 54 (1956) 360-377, p. 365 .

${ }^{3}$ Thiel C. in: J.Speck (Hrsg.), Grundprobleme der großen Philosophen. Philosophie der Gegenwart I, Göttingen 1972, S. 15.
${ }^{4}$ Da Paradoxien häufig und beliebt sind, gibt es dazu sehr viel Literatur. Hier können nur einige Sammeldarstellungen genannt werden, die ihrerseits auf weitere Literatur verweisen. Die schönste $\mathrm{Zu}$ sammenstellung gibt $N$. Falletta, Paradoxon, München 1985. Viele Beispiele und Anregungen bieten aber auch $M$. Gardner, Gotcha! Paradoxien für den Homo ludens, München 1985. - P.Hughes/ G. Brecht, Die Scheinwelt des Paradoxons, Braunschweig 1978. - J.A. Paulos, Ich lache, also bin ich. Einladung zur Philosophie, Frankfurt 1988 (engl. 1985). - R. M. Smullyan, Buch ohne Titel. Eine Sammlung von Paradoxa und Lebensrätseln, Braunschweig 1983 (engl. 1980). - A.K. Suchotin, Kuriositäten in der Wissenschaft, Thun/Frankfurt 1983. - Pragmatische Paradoxien behandeln $P$. Watzlawick u. a., Menschliche Kommunikation, Bern 1969, Kap. 6 und 7. Die logisch-semantischen Antinomien untersucht mit formallogischen Mitteln $F$. v. Kutschera, Die Antinomien der Logik, Freiburg 1964.

${ }_{5}^{5}$ Meschkowski, H. Mathematik als Grundlage, München 1973, Kap. III

${ }^{6}$ Hassenstein. B. Wie viele Körner ergeben einen Haufen? In: A.Peisl/A.Mohler (Hrsg.), Der Mensch und seine Sprache, München 1979, 219 242.

7 Zahlreiche selbstbezügliche Gebilde finden sich bei D.R. Hofstadter in: Spektrum der Wissenschaft März 1981, 6-10.

${ }^{8}$ Der Begriff des „virtuosen Zirkels“ wird eingeführt in G.Vollmer, Was können wir wissen? Band 1: Die Natur der Erkenntnis, Stuttgart 1985, ${ }^{2} 1988$, S. 217-267. 